\title{
OFDM CHANNEL ESTIMATION BY A LINEAR EM-MAP ALGORITHM
}

\author{
J.M Mamfoumbi Ocloo and Florence Alberge \\ Laboratoire des Signaux et Systèmes, CNRS, \\ Plateau de Moulon, 91192 Gif-sur-Yvette cedex, France (Europe) \\ email: $\{$ mamfoumbi, alberge $\} @ 1$ lss.supelec.fr
}

\begin{abstract}
OFDM systems traditionally perform channel coefficients estimation relying on known training sequences. However in wireless systems, performance and mobility can be further enhanced by operating semi-blind channel estimation refinement between reference symbols. A channel tracking method based on a MAP version of the Expectation-Maximization (EM) algorithm and on a block representation of channel variations has been proposed. It performs better than already existing recursive algorithms but have a quadratic complexity. In this paper, we propose a simplified MAP algorithm which has a linear complexity and we examine its convergence properties. This simplification is an adaptation of the One Step Late technique to the EM algorithm for MAP estimation. The proposed algorithm is applicable to both single carrier and OFDM systems. Simulations are presented in the context of $5 \mathrm{GHz}$ WLANs, showing that this algorithm perform as well as the MAP version of the EM algorithm in the mobile environment, without any loss in performance in the static situation.
\end{abstract}

\section{INTRODUCTION}

Orthogonally Frequency Division Multiplexing (OFDM) has already been accepted for Wireless Local Area Network (WLAN) standards (IEEE 802.11a), European Telecommunications Standards Institute's (ETSI) High Performance Local Area Network Type 2 (HIPERLAN 2) and Japan's Mobile Multimedia Access Communication (MMAC) systems. In OFDM, the problem of the intersymbol interference (ISI) is avoided by inserting a cyclic prefix between consecutive transmitted blocks. The effects of the channel appear in the frequency domain as a simple scalar multiplication, each subcarrier being attenuated by the corresponding narrowband subchannel coefficient. Efficient and accurate channel estimation for OFDM is necessary to coherently demodulate received data. Channel parameters estimation has been successfully used to improve the performance of OFDM systems, and plays a crucial role in such systems. Classical methods for estimating these coefficients are based on training sequences. A known sequence is transmitted for a limited period of time, during which a channel estimate is obtained, and a complete description of the transmission system is established. The resulting model is then used for the detection of data symbols subsequently transmitted. In order to cope with Doppler effect due to the mobility of wireless systems, reference sequences must be repeated quite often, and may result in a significant loss in the useful bit rate. An alternative to this scheme is to estimate the channel based only on noisy data exploiting statistical and other properties of the information sequences and channel, this is the blind channel estima- tion approach. A common way to design blind estimation algorithms is to use the Expectation Maximization (EM) algorithm. For likelihood functions with multiple maxima, the convergence point depends of the initial starting point and may be a local maximum. Semi-blind estimation methods consist in tracking the channel variations by refining the channel coefficients blindly using the training sequence as initializations for the estimator, hence local convergence problems are avoided. EM-based blind or semiblind channel estimation methods have already been proposed in the OFDM context [1]-[6], including time and frequency correlations or not. Most proposed methods consider separately each coefficient, and one can obtain improvements by considering blocks of data. In this work, we estimate channel coefficients and channel noise variance simultaneously contrary to precedent methods, which estimate only channel coefficients. We work within the framework of slowly time-varying channels, for which the Doppler speeds are rather small so that inter channel interference (ICI) can be neglected. For such channels, the channel can be considered constant during the transmission of an OFDM symbols block which size depends of the mobility. Based on this idea, a maximum a posteriori (MAP) algorithm which takes into account a channel correlation model between the channel coefficients of different blocks has been proposed in [6]. This prior information permits to improve channel estimation. EM-MAP has a quadratic arithmetic complexity which limits its practical usefulness. In his paper, we propose to reduce this computation complexity. Indeed, using the One Step Late (OSL) algorithm presented in [7] permits to turn the arithmetic complexity from quadratic to linear. The convergence of this new algorithm in our context is established. We also prove that the stationary points are also stationary points of the EM-MAP. As a result, the modified EM-MAP algorithm has the same performances than the EM-MAP with the additional advantage of linear arithmetic complexity.

\section{SYSTEM MODEL}

We consider a conventional cyclic prefix OFDM transceiver scheme depicted in figure 1 , in which a baseband discrete time model of the system is provided. In this model, some side entries of the size $P$ IFFT are zero and only $N c$ subcarriers among the $P$ available are effectively used for transmission of information data. The block of data $\mathbf{x}^{\prime}=\left[x_{0}^{\prime}, \ldots, x_{N_{c-1}}^{\prime}\right]^{t}$ is modulated in the time domain by IFFT. The channel is modeled by linear filtering. Some redundancy is introduced into the transmitted signal by cyclic prefix extension so that the overlapping introduced by the channel memory corresponds to that of a circular convolution between $\mathbf{x}$ and the channel. Consequently, the channel is viewed in the frequency domain after demodulation by the FFT as parallel flat fading channels. Hence, the block $\mathbf{x}$ can easily be retrieved 
from the corresponding received block $\mathbf{y}^{\prime}=\left[y_{1}^{\prime}, \ldots, y_{N c}^{\prime}\right]^{t}$ by FFT and IFFT. Let $\mathbf{h}^{\prime}=\left[h_{0}^{\prime}, \ldots, h_{P-1}^{\prime}\right]^{t}$ denote the OFDM channel, the received signal can be modeled by the following equation :

$$
\mathbf{y}^{\prime}=\operatorname{Diag}\left(\mathbf{x}^{\prime}\right) \mathbf{H}^{\prime}+\mathbf{e}^{\prime}
$$

where $\mathbf{H}^{\prime}=S F \mathbf{h}^{\prime}, F$ is the $P \times P$ Fourier matrix, $S$ is the $N c \times P$ matrix selecting the $N c$ information sub-carriers $S=\left[\mathbf{0}_{\mathbf{N c}, \frac{\mathbf{P}-\mathbf{N c}}{\mathbf{2}}}\right.$

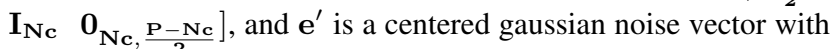
variance $\sigma_{e}^{2}$

Note that $h_{i}^{\prime}=0$ for $i \geq L$, where $L$ denotes the cyclic prefix length. From now on, vectors $\mathbf{x}^{\prime}, \mathbf{y}^{\prime}$ and $\mathbf{H}^{\prime}$ denote respectively the data vector, the observation vector and the channel coefficient vector for a given OFDM symbol. The OFDM systems are designed such that $L<P$ (in IEEE802.11a, $L=P / 4$ ). The taps are assumed independent and Rayleigh distributed.

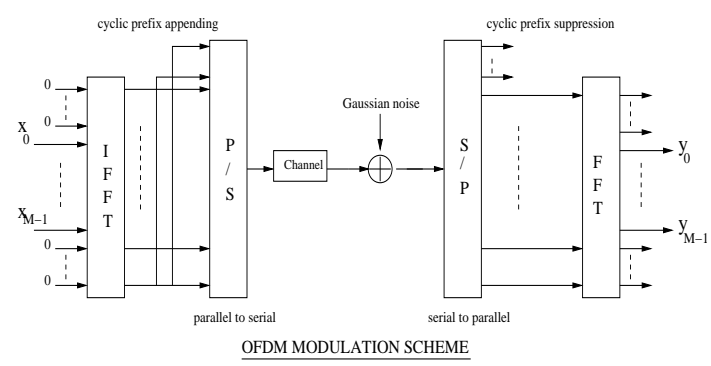

Fig. 1. Conventional OFDM Transceiver

\section{EM-MAP ALGORITHM}

Since diversity improves performances of ML or MAP estimators, a channel model which is piecewise constant has been proposed in [5] : it has the same channel coefficient for the transmission of several consecutive symbols. The application of the MAP version of the EM algorithm to this channel model made it possible to improve considerably the performances in term of channel estimation. First, the EM-MAP algorithm is quickly reviewed.

Assume that the channel coefficient $H_{k}$ can be modeled as the output of a stationary autoregressive (AR) process of order 1 , namely : $H_{k}=\alpha H_{k-1}+\varepsilon_{k}$, where $\alpha$ is the time correlation coefficient and $\varepsilon_{k}$ is an additive white gaussian noise of mean 0 and variance $\sigma_{\varepsilon}^{2} . H_{k}$ denotes the channel coefficient of the block $k$ and is supposed constant over a block of length T. The parameter $\sigma_{\varepsilon}^{2}$ is unknown and will be estimated by the proposed procedure. On another side, $\alpha$ is assumed to be known. Indeed, $\alpha$ can be perfectly determined when the Doppler spread $B_{d}$ and the delay spread $T_{m}$ are known thanks to Jakes' model :

$$
\Phi(\Delta f, \Delta t)=\Phi(0,0) \frac{J 0\left(\pi B_{d} \Delta t\right)}{1+j 2 \pi T_{m} \Delta f}
$$

where $\Phi(\Delta f, \Delta t)$ is the channel time-frequency covariance function for a classical Doppler power spectrum and an exponential multipath intensity profile with mean power $\Phi(0,0)$ and $J 0$ is the zero-order Bessel function of the first kind.

Let $\tilde{\alpha}\left(\tilde{\alpha}=\Phi(0,0) J 0\left(\pi B_{d} \Delta t\right)\right)$ denote the correlation coefficient for the true channel, then the correlation coefficient $\alpha$ (used in the block model) is set to $\alpha=(\tilde{\alpha})^{T}$, where T denotes the block size. The EM algorithm has been easily modified to produce the MAP estimate. Using matrix notations, one has obtained for a given subcarrier :

$$
\begin{aligned}
& \mathbf{y}=\mathbf{X H}+\mathbf{e} \\
& \mathbf{M H}=\varepsilon+\mathbf{b}
\end{aligned}
$$

where $\mathbf{M}$ is a $q \times q$ matrix given by :

$$
\mathbf{M}=\left(\begin{array}{ccccc}
1 & 0 & \cdots & \cdots & 0 \\
-\alpha & 1 & \ddots & \ddots & \vdots \\
0 & \ddots & \ddots & \ddots & \vdots \\
\vdots & \ddots & \ddots & \ddots & 0 \\
0 & \cdots & 0 & -\alpha & 1
\end{array}\right)
$$

The auxiliary function : $Q\left(\mathbf{H}, \sigma_{e}^{2}, \sigma_{\varepsilon}^{2}, \mathbf{H}^{(i)}, \sigma_{e}^{2(i)}, \sigma_{\varepsilon}^{2(i)}\right)$

$$
\begin{aligned}
=\sum_{\underline{m}} P\left(\mathbf{S}_{\underline{m}} \mid \mathbf{y}, \mathbf{H}^{(i)}, \sigma_{e}^{2(i)}\right) & {\left[\log P\left(\mathbf{y} \mid \mathbf{S}_{\underline{m}}, \mathbf{H} ; \sigma_{e}^{2}\right)\right.} \\
+ & \left.\log P\left(\mathbf{H}, \sigma_{\varepsilon}^{2}\right)+c t e\right]
\end{aligned}
$$

with $P\left(\mathbf{y}, \mathbf{S}_{\underline{m}} \mid \mathbf{H}, \frac{1}{\sigma_{e}^{2}}\right)=P\left(\mathbf{S}_{\underline{m}}\right) \frac{1}{\sigma_{e}^{2 N}} \exp \left(-\frac{1}{\sigma_{e}^{2}}\left\|\mathbf{y}-\mathbf{S}_{\underline{m}} \mathbf{B H}\right\|^{2}\right)$ and $P\left(\mathbf{H}, \sigma_{\varepsilon}^{2}\right) \propto \frac{1}{\sigma_{\varepsilon}^{2 q}} \exp \left(-\frac{1}{\sigma_{\varepsilon}^{2}}\left(\mathbf{H}-\mathbf{M}^{-1} \mathbf{b}\right)^{*} \mathbf{C}^{-1}\left(\mathbf{H}-\mathbf{M}^{-1} \mathbf{b}\right)\right)$ where $\sigma_{\varepsilon}^{2} \mathbf{C}$ is the covariance matrix of $\mathbf{H}$. By setting to zero the derivative of $Q\left(\mathbf{H}, \sigma_{e}^{2}, \sigma_{\varepsilon}^{2}, \mathbf{H}^{(i)}, \sigma_{e}^{2(i)}, \sigma_{\varepsilon}^{2(i)}\right)$ with respect to $\mathbf{H}^{*}$, we obtain :

$$
\left[\frac{1}{\sigma_{e}^{2}} \frac{\mathbf{D}}{\Gamma}+\frac{1}{\sigma_{\varepsilon}^{2}} \mathbf{C}^{-1}\right] \mathbf{H}^{(i+1)}=\frac{1}{\sigma_{e}^{2}} \frac{\mathbf{V}}{\Gamma}+\frac{1}{\sigma_{\varepsilon}^{2}} \mathbf{M}^{*} \mathbf{b}
$$

where matrix $\mathbf{D}$, vector $\mathbf{V}$ and $\Gamma$ are given by :

$$
\begin{aligned}
\mathbf{D} & =\sum_{\underline{m}} P\left(\mathbf{S}_{\underline{m}} \mid \mathbf{y}, \mathbf{H}^{(i)}, \sigma_{e}^{2(i)}\right) \mathbf{B}^{*} \mathbf{S}_{\underline{m}}^{*} \mathbf{S}_{\underline{m}} \mathbf{B} \\
\mathbf{V} & =\sum_{\underline{m}} P\left(\mathbf{S}_{\underline{m}} \mid \mathbf{y}, \mathbf{H}^{(i)}, \sigma_{e}^{2(i)}\right) \mathbf{B}^{*} \mathbf{S}_{\underline{m}}^{*} \mathbf{y} \\
\Gamma & =\sum_{\underline{m}} P\left(\mathbf{S}_{\underline{m}} \mid \mathbf{y}, \mathbf{H}^{(i)}, \sigma_{n}^{2(i)}\right)
\end{aligned}
$$

The the current channel estimates are obtained by solving the linear system (5) by a Gauss method. This step has a quadratic arithmetic complexity.

By similar calculations, we obtain the update formulae of the channel noise variance and of the noise variance which have a linear arithmetical complexity.

\section{SIMPLIFIED EM-MAP}

Two main points are very important in the design of a good channel estimator, namely : the number of pilot symbols which must be as small as possible and the estimator arithmetic complexity which must also be as small as possible. EM-MAP algorithm realizes a good estimation but its complexity is still important. We propose an alternative to reduce arithmetic complexity while keeping good performances in term of channel estimation and bit error rate.

\subsection{One Step Late (OSL) algorithm}

The methodology of the EM algorithm consists, by a judicious choice, in reformulating the incomplete-data problem by completedata problem. This algorithm usually gives explicit expressions for the parameter $\Theta$ maximizing the auxiliary function $Q\left(\Theta, \Theta^{(i)}\right)$. But in many situations, the corresponding update obtained is far more complicated. This is particularly likely to be so if the prior probability term represents a roughness penalty, when each component of the derivative typically involves several components of 
$\Theta$. In our case, however, it is possible to modify this algorithm quite simply, in order to avoid this difficulty. After each update process, replace $\Theta^{(i)}$ by the new parameter which maximizes the current auxiliary function. The main idea consists in computing the derivative of the prior probability at the current value of $\Theta$, rather than at the new value. An intuitive justification of this procedure is that if the algorithm converges slowly, the derivative computed at $\Theta^{(i)}$ and $\Theta^{(i+1)}$ will not be much different [7]. Moreover, it can straightforwardly be seen that this method has exactly the same fixed points than the original algorithm. In the general case however, the OSL algorithms are not guaranteed to converge neither to increase the penalized log-likelihood. We prove in section 4.3 below that the proposed algorithm converges and increases the penalized log-likelihood.

\subsection{Algorithm description}

From (3), we see that the log-probability of $\mathbf{H}$ reads :

$\log (p(\mathbf{H}))=-\frac{1}{\sigma_{\varepsilon}^{2}}\left(\mathbf{H}-\mathbf{M}^{-1} \mathbf{b}\right)^{*} \mathbf{C}^{-1}\left(\mathbf{H}-\mathbf{M}^{-1} \mathbf{b}\right)-q \log \left(\sigma_{\varepsilon}^{2}\right)$

The quadratic complexity of the EM-MAP algorithm is due to the quadratic term $-\frac{1}{\sigma_{\varepsilon}^{2}} \mathbf{H}^{*} \mathbf{C}^{-1} \mathbf{H}$ since $\mathbf{C}^{-1}$ is not a diagonal matrix. Following the One Step late (OSL) technique, we replace the gradient of $-\frac{1}{\sigma_{\varepsilon}^{2}} \mathbf{H}^{*} \mathbf{C}^{-1} \mathbf{H}$ with respect to $\mathbf{H}^{*}$ by the gradient computed at $\mathbf{H}=\mathbf{H}^{(i)}$. An analogous expression could have also been obtained by replacing $p(\mathbf{H})$ with $p^{\prime}(\mathbf{H})$ defined as :

$$
\begin{array}{r}
\log \left(p^{\prime}(\mathbf{H})\right)=-\frac{1}{\sigma_{\varepsilon}^{2}}\left[\left(\mathbf{H}-\mathbf{M}^{-1} \mathbf{b}\right)^{*} \mathbf{C}^{-1}\left(\mathbf{H}-\mathbf{M}^{-1} \mathbf{b}\right)\right. \\
\left.-\left(\mathbf{H}-\mathbf{H}^{(i)}\right)^{*} \mathbf{C}^{-1}\left(\mathbf{H}-\mathbf{H}^{(i)}\right)\right]-q \log \left(\sigma_{\varepsilon}^{2}\right)
\end{array}
$$

However, we prefer to consider a new prior $p^{\prime \prime}(\mathbf{H})$ such that :

$$
\log \left(p^{\prime \prime}(\mathbf{H})\right)=\log \left(p^{\prime}(\mathbf{H})\right)-\frac{\beta}{\sigma_{\varepsilon}^{2}}\left(\mathbf{H}-\mathbf{H}^{(i)}\right)^{*}\left(\mathbf{H}-\mathbf{H}^{(i)}\right)
$$

where the second term is introduced to ensure the convergence of the method. This point will be addressed in the next section where we will also explain how to choose the value of the parameter $\beta$. With this new prior $p^{\prime \prime}(\mathbf{H})$, the update equation for the channel parameter is given by :

$$
\begin{aligned}
& {\left[\frac{1}{\sigma_{e}^{2}} \frac{\mathbf{D}}{\Gamma}+\frac{\beta}{\sigma_{\varepsilon}^{2}} I\right] \mathbf{H}^{(i+1)}=\frac{1}{\sigma_{e}^{2}} \frac{\mathbf{V}}{\Gamma}+\frac{1}{\sigma_{\varepsilon}^{2}} M^{*} \mathbf{b}} \\
& -\frac{1}{\sigma_{\varepsilon}^{2}} \mathbf{C}^{-1} \mathbf{H}^{(i)}+\frac{\beta}{\sigma_{\varepsilon}^{2}} \mathbf{H}^{(i)}
\end{aligned}
$$

where $\mathbf{D}, \mathbf{V}, \Gamma$ are defined in section 3 . The update equation for the variances $\sigma_{e}^{2}$ and $\sigma_{\varepsilon}^{2}$ are unchanged. We can remark that $\mathbf{H}^{(i+1)}$ is multiplied by a diagonal matrix. The computational complexity of this step is then linear [5].

Moreover, when this algorithm converges, the fixed points are the fixed points of the EM-MAP.

\subsection{Convergence study}

It was recently shown that the EM algorithm can be viewed as a particular case of a PPA (Proximal Point Algorithm) [8]. An elegant way to prove the convergence of the EM algorithm is to consider it as a particular case of PPA algorithm. We rely on these results to establish the convergence of our modified algorithm.

\subsubsection{Convergence of the PPA and link with the EM-MAP algo-} rithm

A generalized PPA type algorithm is defined by the iterative process [8] :

$$
\hat{\Theta}^{(i+1)}=\arg \max _{\Theta}\left\{\epsilon(\Theta)-\beta_{i} d\left(\Theta, \widehat{\Theta}^{(i)}\right)\right\}
$$

where $\beta_{i}$ is a sequence of positive number and $d\left(\Theta, \widehat{\Theta}^{(i)}\right)$ is a penalty function such that :

$$
\begin{aligned}
& d\left(\Theta, \widehat{\Theta}^{(i)}\right) \geq 0 \\
& d\left(\Theta, \widehat{\Theta}^{(i)}\right)=0 \quad \text { if and only if } \Theta=\widehat{\Theta}^{(i)}
\end{aligned}
$$

The original PPA with $d\left(\Theta, \widehat{\Theta}^{(i)}\right)=\left\|\Theta-\widehat{\Theta}^{(i)}\right\|^{2}$ was proposed and studied in [4]. One can easily show that $\left\{\epsilon\left(\Theta^{(i))}, i=\right.\right.$ $0,1,2 \ldots\}$ is a nondecreasing sequence [8], meaning that :

$$
\epsilon\left(\widehat{\Theta}^{(i+1)}\right)-\epsilon\left(\widehat{\Theta}^{(i)}\right) \geq 0 \forall i \in \mathbb{N}
$$

It turns out that the EM-MAP is a PPA with $\epsilon(\Theta)=\log p(y \mid \Theta)+$ $\log (\Theta), \beta_{i}=1$ and

$$
\begin{aligned}
d\left(\Theta, \widehat{\Theta}^{(i)}\right) & =\mathcal{D}_{K L} \\
& =\left[p\left(x \mid y, \Theta^{(i)}\right) \| p(x \mid y, \Theta)\right] \\
& =\int p\left(x \mid y, \widehat{\Theta}^{(i)}\right) \log \frac{p\left(x \mid y, \widehat{\Theta}^{(i)}\right)}{p(x \mid y, \Theta)} d x \\
& =E\left[\log \frac{p\left(x \mid y, \widehat{\Theta}^{(i)}\right)}{p(x \mid y, \Theta)} \mid x, \widehat{\Theta^{(i)}}\right]
\end{aligned}
$$

Since $d\left(\Theta, \widehat{\Theta}^{(i)}\right)$ is a Kullback-Liebler (KL) divergence, both conditions $d\left(\Theta, \widehat{\Theta}^{(i)}\right) \geq 0$ and $d\left(\Theta, \widehat{\Theta}^{(i)}\right)=0$ if and only if $\Theta=$ $\widehat{\Theta^{(i)}}$ are verified. Moreover $\epsilon(\Theta)$ appears to be the a posteriori log-probability. Thus the EM-MAP algorithm increases (or keep constant) the a posteriori probability(APP) when the number of iterations increases. Consequently, the EM-MAP algorithm converges to a stationary point of the APP. Obviously, this is not a new result but this method is well suited for studying the convergence of the proposed algorithm.

\subsubsection{Modified EM-MAP convergence}

The modification introduced by the OSL-like method consists in replacing the prior $\log (p(\mathbf{H}))$ by $\log \left(p^{\prime \prime}(\mathbf{H})\right)$. The relationship between $\log (p(\mathbf{H}))$ and $\log \left(p^{\prime \prime}(\mathbf{H})\right)$ can be expressed as :

$\log \left(p^{\prime}(\mathbf{H})\right)=\log (p(\mathbf{H}))+\frac{1}{\sigma_{\varepsilon}^{2}}\left(\mathbf{H}-\mathbf{H}^{(i)}\right)^{*}\left(C^{-1}-\beta I\right)\left(\mathbf{H}-\mathbf{H}^{(i)}\right)$

This new algorithm is a PPA defined by the iteration :

$$
\widehat{\Theta}^{(i+1)}=\arg \max _{\Theta}\left(\varepsilon(\Theta)-\beta_{i} d^{\prime}\left(\Theta, \widehat{\Theta}^{(i)}\right)\right)
$$

where $\varepsilon(\Theta)$ is still the a posteriori log-probability and $d^{\prime}\left(\Theta, \Theta^{(i)}\right)=$ $d\left(\Theta, \Theta^{(i)}\right)+\frac{1}{\sigma_{e}^{2}}\left(\mathbf{H}-\mathbf{H}^{(i)}\right)^{*}\left(\beta I-C^{-1}\right)\left(\mathbf{H}-\mathbf{H}^{(i)}\right)$ and $\beta_{i}=1$. The matrix $\beta I-C^{-1}$ must be a positive definite matrix to satisfy :

$$
\left\{\begin{array}{l}
d^{\prime}\left(\Theta, \widehat{\Theta}^{(i)}\right) \geq 0 \\
d^{\prime}\left(\Theta, \widehat{\Theta}^{(i)}\right)=0 \quad \text { if and only if } \Theta=\widehat{\Theta}^{(i)}
\end{array}\right.
$$




\begin{tabular}{||c||c|c||}
\hline & EM-MAP $(0 \mathrm{~m} / \mathrm{s})$ & EM-OSL $(0 \mathrm{~m} / \mathrm{s})$ \\
\hline \hline $\mathbf{1 0} \mathbf{~ d B}$ & $2.6875 \mathrm{e}-2$ & $2.392 \mathrm{e}-2$ \\
\hline $\mathbf{1 2} \mathbf{~ d B}$ & $5.5539 \mathrm{e}-3$ & $4.8848 \mathrm{e}-3$ \\
\hline
\end{tabular}

Table 1. BER comparison for a static bran c channel

\begin{tabular}{||c||c|c||}
\hline & EM-MAP $(3 \mathrm{~m} / \mathrm{s})$ & EM-OSL $(3 \mathrm{~m} / \mathrm{s})$ \\
\hline \hline $\mathbf{1 0} \mathbf{~ d B}$ & $3.6567 \mathrm{e}-2$ & $3.046 \mathrm{e}-2$ \\
\hline $\mathbf{1 2} \mathbf{~ d B}$ & $7.0767 \mathrm{e}-3$ & $7.8444 \mathrm{e}-3$ \\
\hline
\end{tabular}

Table 2. BER comparison for a bran c channel with a velocity of $3 \mathrm{~m} / \mathrm{s}$

By providing a majoration of the Rayleigh quotient of the hermitian matrix $\mathbf{C}^{-1}$, we prove that all the eigenvalues of $\mathbf{C}^{-1}$ are less or equal to $(1+\alpha)^{2}$. We then choose $\beta>(1+\alpha)^{2}$. Note that the value of $\beta$ does not appear in the expression of the fixed point. However, this parameter may have some influence on the speed of convergence of the method but we did not elaborate on this matter for the moment. In conclusion, the proposed method has exactly the same fixed points than the original EM-MAP algorithm, the a posteriori probability increases (or at least does not decrease) with the iterations provided that $\beta>(1+\alpha)^{2}$ and the computational complexity is linear.

\section{SIMULATIONS}

Simulations have been performed in the specific context of HIPERLAN/2 broadband wireless communication standard, which is similar to IEEE802.11a and MMAC. HIPERLAN/2 is a system operating at the $5 \mathrm{GHz}$ and using a $20 \mathrm{MHz}$ bandwidth at typical SNR value of $0-20 \mathrm{~dB}$ for slowly terminal speeds. The cyclic prefix is 16 samples long and the number of carriers is $N_{c}=64$. A rate $R=1 / 2$, constraint length $l=7$ Convolutional Code (CC) $(171 / 133)$ is used before bit interleaving followed by 16-QAM mapping. Only 48 carriers are effectively used. Monte Carlo simulations are run and averaged over 5000 realizations of a BRAN $\mathrm{C}$ frequency selective channel in order to obtain BER curves. A classical Jake's Doppler spectrum and Rayleigh fading statistics are assumed for all taps. Results are provided both for static channel and for time-varying channels for several terminal speeds, as specified in the HIPERLAN/2 standard. These speeds are supposed to be known from the receiver. Each frame processed contains 2 known training symbols, followed by 100 OFDM data symbols. The bit probabilities $P\left(b_{l}^{m}\right)$ estimates are performed in the E-step by two iterations of the turbo demodulation process, which is an iterative joint demapping algorithm yielding a better estimation of $P\left(b_{l}^{m}\right)$ than classical methods like BJCR algorithm. The block size is set to $T=20$ for Doppler speeds less than $3 \mathrm{~m} / \mathrm{s}$ and to $T=10$ for speeds higher than $3 \mathrm{~m} / \mathrm{s}$. We choose $\beta=2\left(1+\alpha^{2}\right)$. The estimation process is repeated until the mean square errors of the channel coefficients matrix and the channel noise variance are lower than $10^{-8}$ simultaneously. We compare performances in term of channel estimators between the EM-Block [5], the EM-MAP and the EM-OSL (the proposed algorithm) algorithms. Figure 2 depicts the mean square error as a function of SNR. It appears clearly that the simplified MAP algorithm, which has a linear complexity, performs better than the EM-Block algorithm and has the same performances that the initial EM-MAP algorithm. Tables 1 and 2 confirm this results in term of BER.

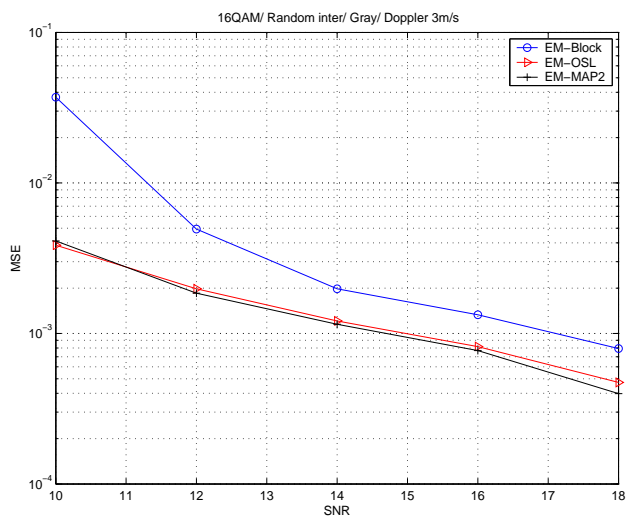

Fig. 2. MSE for channel with a velocity of $3 \mathrm{~m} / \mathrm{s}$

\section{CONCLUSION}

This paper has presented a new blind channel estimation concept for OFDM systems. Making use of the diversity introduced by the fact that, for slowly time-varying channels the channel coefficient does not change quickly from one symbol to another. This idea has been used to develop three algorithms based on the EM algorithm. The direct application of a block model showed improved performance of the corresponding MAP version. This version still improves the performances in terms of channel estimation or BER, however the corresponding complexity is important. We then proposed a simplified version inspired by the OSL algorithm which has a linear complexity and performs as well as the MAP algorithm. Further work consists in taking into account a time-frequency model of the channel variations in the EM Block procedure in order to still improve the performances. All of these methods can also be used in the MIMO-OFDM context, where several works already focused on the EM algorithm to perform channel estimation.

\section{ACKNOWLEDGEMENT}

The authors are grateful to Professor Pierre Duhamel who is co-author and whose the name cannot appear on this paper because it already appears on 5 others.

\section{REFERENCES}

[1] E. Jaffrot and M. Siala, "Turbo channel estimation for OFDM systems on highly time and frequency selective channels" in IEEE International Conference on Acoustics, Speech, and Signal Processing, Istanbul, Turkey, June rence 2000.

[2] X. Zhuang and F. Vook, "Iterative channel estimation and decoding for a turbocoded OFDM system via the EM algorithm", Proc 56th IEEE VTC 2002.

[3] Tareq Y. Al-Naffouri, Ahmad Bahai and Arogyaswami Paulraj, "An EMbased OFDM receiver for time-variant channels", Global Telecommunications Conference, 2002. GLOBECOM '02. IEEE Volume 1, 17-21 Nov. 2002 Page(s) :589 - 593 vol.1

[4] B. Martinet, "Régularisation d'inéquations variationnelles par approximations successives," Rev. Française d'inform. et de Rech. Operationnelle, vol. 3, pp 154-179, 1970

[5] S. Touati, JM. Mamfoumbi-Ocloo, F. Alberge and P. Duhamel, "Semi-Blind channel estimation for OFDM systems via an EM-Block algorithm", EUSIPCO, Vienna, September 2004.

[6] JM. Mamfoumbi-Ocloo, F. Alberge and P. Duhamel, "Semi-Blind channel estimation for OFDM systems via an EM-MAP algorithm", SPAWC 2005, 6th IEEE workshop, june 2005

[7] P.J Green, "On the use of the EM algorithm for penalized likelihood estimation", J.R statist. Soc B, 52(3) :443-452, 1990.

[8] Stéphane Chrétien and Alfred O. Hero III, "Kullback Proximal Algorithms for Maximum-Likelihood Estimation", IEEE transactions on information theory, vol. 46, N0.5, August 2000. 